livraisons

d'Histoire

de l'Architecture

\section{Livraisons de l'histoire de l'architecture}

39 | 2020

Maquettes d'architecture

\title{
Entre expérimentation, démonstration et transmission : la maquette dans le contexte de la charpenterie traditionnelle au Japon
}

Between experimentation, demonstration and transmission: The model in the context of traditional carpentry in Japan

Zwischen Erforschung, Vorführung und Weitergabe: Das Architekturmodell im Kontext des traditionellen Zimmerhandwerks in Japan

\section{Soizik Bechetoille-Kaczorowski}

\section{OpenEdition \\ Journals}

Édition électronique

URL : http://journals.openedition.org//ha/1602

DOI : 10.4000/lha.1602

ISSN : 1960-5994

Éditeur

Association Livraisons d'histoire de l'architecture - LHA

Édition imprimée

Date de publication : 15 juin 2020

Pagination : 115-124

ISSN : 1627-4970

Référence électronique

Soizik Bechetoille-Kaczorowski, «Entre expérimentation, démonstration et transmission : la maquette dans le contexte de la charpenterie traditionnelle au Japon », Livraisons de l'histoire de l'architecture [En ligne], 39 | 2020, mis en ligne le 24 janvier 2021, consulté le 26 janvier 2021. URL : http:// journals.openedition.org/lha/1602 ; DOI : https://doi.org/10.4000/lha.1602 


\section{ENTRE EXPÉRIMENTATION, DÉMONSTRATION ET TRANSMISSION : LA MAQUETTE DANS LE CONTEXTE DE LA CHARPENTERIE TRADITIONNELLE AU JAPON}

Dans le contexte de la conservation de bâtiments, hypothèses, projections, compromis, renoncements, sont autant d'éléments qui fondent les choix architecturaux et patrimoniaux. Entre la nécessité de comprendre pour soi-même ou pour le groupe d'opérateurs (architectes, charpentiers, artisans...) et le besoin de communiquer à un public plus large, la maquette en volume est un outil au service du projet, en particulier au Japon où elle tient plusieurs rôles dans un chantier de restauration. Dans le cadre des chantiers de démontage périodique, elle présente des détails de structure complexes et illustre certains aspects des travaux effectués. Elle est réalisée avec les matériaux, les assemblages et les outils de prédilection du charpentier, et permet aux apprentis daiku-san 大工さん (charpentiers), mais aussi au public et aux commanditaires de comprendre certaines difficultés techniques. Véritable support de dialogue, elle est projet en tant que tel. Les acteurs des travaux peuvent la produire pour vérifier certaines hypothèses relatives, par exemple, à l'agencement (ordre d'assemblage), mais une fois fabriquée, la maquette qui a permis aux charpentiers d'anticiper leurs opérations, peut encore communiquer ces aspects aux autres, ce qui amorti de manière significative l'investissement qu'a représenté sa fabrication. Tout est signifiant dans une maquette de chantier : son échelle, ses matériaux, sa facture, ses finitions. S'il existe plusieurs raisons de la produire durant le chantier, nous évoquerons les cas particuliers de la restauration par démontage périodique et de la reconstruction qui permettent d'aborder différentes fonctions de la maquette à travers trois exemples allant de la simple vérification d'hypothèses techniques à la fonction didactique élargie.

\section{La maquette de La pagode à cinq étages (Gojūnotō 五重塔), nouvelle de l'écrivain Kōda Rohan - $1892^{1}$}

L'histoire de La pagode à cinq étages, qui relate la façon dont un charpentier de condition modeste nommé Jūbei 十兵衛 se persuada qu'il devait construire une pagode à cinq étages, nous permet de comprendre un premier usage de la maquette

1. Kōda Rohan, La Pagode à cinq étages, et autres récits, Paris, Les Belles Lettres, collection "Japon ", 2009. 
de chantier. Le prêtre bouddhiste à l'origine de la commande voulait utiliser des fonds restants de la rénovation du temple. Alors que le chantier revenait de droit au maître charpentier Genta Kawagoe 川越の源太, l'artisan Jūbei outrepassa son rang, força les carcans rigides de la société et présenta la maquette de son projet directement au prêtre. Après examen de ladite maquette, le religieux, qui dit avoir vu à travers cet objet " la technique, la fougue et le malheur ", reconnut les qualités architecturales du petit artisan. Le prêtre convoqua donc le maître charpentier et son apprenti et leur demanda de nommer par eux-mêmes le responsable du chantier, car lui ne pouvait choisir entre expérience et passion. Genta proposa une collaboration, mais Jūbei refusa. Le prêtre donna finalement à Jūbei la pleine autorité sur le projet. Une fois la pagode achevée, elle fut frappée par un terrible orage. Alors que tout le monde craignait qu'elle ne s'écroule, elle resta vaillamment érigée. Dans les jours qui suivirent, l'abbé consacra la pagode par une inscription où l'on pouvait lire, entre autres, le nom de l'artisan Jūbei.

Cette nouvelle de l'écrivain Kōda Rohan 幸田 露伴2, écrite à l'origine comme une série de fiction dans le journal Kokkai 国会新聞 de novembre 1891 à mars 1892, est inspirée de la première pagode à cinq étages de Yanaka 谷中 qui se trouvait dans l'enceinte du temple Gokokuzan Soncho.in Tenno-ji 護国山尊重院天王寺 ${ }^{3}$ de la secte Tendai 天台宗. Véritable prouesse technique permettant à Rohan de souligner l'audace et l'habileté de celui qui parvint à l'ériger, la première pagode à cinq étages, construite en 1644, disparut dans un incendie près de 130 ans plus tard, en 1772. Reconstruit 19 ans après la catastrophe, le bâtiment a longtemps servi de repère à Yanaka et, s'il avait échappé aux dégâts causés par les tremblements de terre et les raids aériens de la Seconde Guerre mondiale, il fut à nouveau incendié le 6 juillet 1957. La pagode originale était entièrement construite en bois de zelkova japonais et mesurait 34,18 mètres de haut, ce qui faisait d'elle la plus haute des pagodes bouddhistes de la région du Kantō 関東.

Dans le récit de Rohan, la maquette est présentée comme l'élément permettant au simple charpentier de devenir chef de chantier. Si elle offre au maître d'ouvrage de visualiser le bâtiment achevé, elle le rassure également sur les compétences techniques de l'artisan qui la soumet. En effet, une des spécificités des maquettes produites dans le contexte de la charpenterie traditionnelle japonaise est qu'elles sont nécessairement réalisées dans le respect de l'art du kiwari 梆 ${ }^{4}$ (stéréotomie du bois), c'est-à-dire sans clou ni colle : avec une attention toute aussi élevée pour l'apparence extérieure que pour la recherche des modes d'assemblage qui seront utilisés sur le chantier grandeur nature. Les emboîtements, mêmes complexes, sont réalisés au millimètre près, selon les techniques transmises à travers les siècles et au moyen d'outils eux-mêmes confectionnés dans une échelle adaptée à l'ouvrage.

2. Kōda Rohan, né le 23 juillet 1867 à Shitaya, Tokyo, mort le 30 juillet 1947 à Ichikawa, est le nom d'auteur de l'écrivain japonais Kōda Shigeyuki 幸田成行.

3. Le temple a aujourd'hui fait place à l'actuel cimetière de Yanaka.

4. Voir Philippe Bonnin et Masatsugu Nishida, "Kiwari 木割 - la stéréotomie du bois », Philippe Bonnin, Masatsugu Nishida et Inaga Shigemi (dir.), Vocabulaire encyclopédique de la spatialité japonaise, Paris, CNRS, p. 257-258. 
Cette particularité confere à la maquette un quasi statut de "double miniature " de l'édifice. Les mots pour désigner la maquette en japonais, mokei 模型 et hinagata 鶵型, renvoient effectivement à l'idée de miniature ou de modèle réduit d'une œuvre d'art planifiée, identique dans sa forme et ses proportions à l'objet final dont elle sert de modèle préparatoire. Il semble que les hinagata sont utilisés au Japon depuis l'époque Asuka (fin du VI ${ }^{e}$, début du VIII ${ }^{e}$ siècle). Pour comprendre l'importance particulière accordée à la maquette, il faut bien sûr considérer l'importance endémique de la culture du bois, matériau commun à la miniature et au bâtiment fini.

«En dépit des dommages souvent sérieux provoqués par le feu, les typhons et les tremblements de terre, l'architecture japonaise a donc presque toujours eu pour matériau principal le bois. Le fatalisme, ou plutôt ce sentiment qui fait considérer la maison comme une chose de nature temporaire et transitoire, s'est maintenu depuis le Moyen Âge ${ }^{5}$ ".

Ce caractère transitoire que décrit Masuda Tomoya 増田友也 ${ }^{6}$ (1914-1981) est lié au matériau même. De ce phénomène naît l'idée d'impermanence (mujō 無常), et avec elle une des particularités des techniques relatives à la transmission de l'architecture au Japon: le démontage périodique des édifices. Dans ce contexte particulier où les matériaux qui composent un bâtiment ne sont pas toujours capables d'assurer sa longévité, la maquette intervient à la fois comme objet de conservation (pour celui qui en hérite) et de compréhension (pour celui qui la produit).

\section{La reconstruction de la porte Suzaku 朱排 de 1993 et sa maquette de 1965}

De nombreux travaux de reconstruction ont été menés au Japon depuis la période moderne. Dans la plupart des cas, ces travaux s'appuient sur des bases scientifiques, mais la portée et les raisons qui mènent au choix d'une reconstruction varient d'un objet à l'autre. L'entretien et les révisions nécessaires à la continuité des bâtiments dans le temps lient inévitablement ceux-ci à l'époque de leur construction. Un bâtiment démonté, transporté à un autre endroit, puis remonté, garde une continuité d'usage. Il maintient surtout une vérité intrinsèque, qu'il ait été déplacé, reconstruit, ou qu'on y ait ajouté des éléments. Selon Yagasaki Zentarō, cette pratique est un vecteur de préservation et de transmission des techniques de charpenterie :

5. Masuda Tomoya 増田友也, Japon, Fribourg, Office du livre, coll. "Architecture universelle", 1969, p. 119.

6. Architecte du courant moderniste et chercheur, Masuda Tomoya a notamment introduit l'anthropologie architecturale au Japon. Diplômé de l'université de Kyōto en 1939, il y enseigne jusqu'en 1978.

7. Voir Murielle Hladik, «Mujo 無常 - l'impermanence », Philippe Bonnin, Masatsugu Nishida, Inaga Shigemi (dir.), op. cit., p. 356-358. 
"Pour apprécier la valeur de l'architecture japonaise, il est [ \&] essentiel de prendre en compte ce qui est intangible - les techniques de charpenteries et les éléments tangibles - les structures. [...] La valeur effective de l'architecture de bois au Japon est donc attachée à l'habileté des charpentiers, à leur capacité à transformer, voire à effacer les édifices temporairement, tout en étant à même de ramener à la vie la forme originelle d'un bâtiment disparu. De cette façon, l'architecture japonaise thésaurise de l'histoire, consolide sa valeur historique par la répétition séquentielle de déplacement et d'ajouts, de reconstructions. Dans bien des cas, la date et la forme exactes de la construction d'origine sont floues, puisque le bâtiment a pu être démonté puis reconstruit de nombreuses fois. C'est en ce sens que l'architecture japonaise présente un caractère transitoire ${ }^{8}$."

Au Japon, il est communément admis qu'une des principales caractéristiques des vestiges archéologiques réside dans l'absence de ruine. De fait, la construction en bois peut être aisément démontée, reconstruite, démantelée, partiellement réutilisée, détruite ou abandonnée. Avec le temps, elle se dégrade et disparaît complètement par pourrissement. Comme les traces enfouies dans le sol demeurent extrêmement fragiles, les sites historiques présentent ce caractère particulier où il reste très peu de vestiges sur le terrain - quelques "trous " laissés dans le sol, une "photographie en négatif" des bases de bâtiments. De plus, il est extrêmement difficile de montrer des vestiges en bois très fragiles, une fois ceux-ci exposés à l'air. Les précautions à prendre en termes de conservation sont innombrables et concernent, entre autres, le maintien dans un environnement identique. Aussi, les éléments authentiques ne peuvent guère être observés in situ dans les périmètres historiques. La fugacité des vestiges a donc contribué à la formation d'une culture de tradition littéraire au Japon, où l'apparence passée de sites anciens est largement nourrie d'aspirations imaginaires.

La position et la taille de la porte Suzaku ${ }^{9}$ du site palatial d'Heijō, qui correspond aujourd'hui à la ville de Nara 奈良 (région du Kansai, capitale du Japon au VIII ${ }^{e}$ siècle) ont été déterminées par des fouilles menées en 1964. Celles-ci ont révélé que la distance entre chaque poteau était de 17 shaku ${ }^{10}$ (environ 5 mètres), et que le bâtiment comptait 5 travées de long (environ 25 mètres) pour une profondeur de 2 travées (environ 10 mètres). Les fondations de la plate-forme ont été remplies de terre battue et des pierres retrouvées sur le site ont été ajoutées aux soubassements. Les tuiles retrouvées lors des fouilles ont suggéré qu'elles étaient,

8. Yagasaki Zentarō, "Le caractère transitoire de l'architecture japonaise: déplacements, ajouts, reconstructions ", Jean-Sébastien Cluzel et Nishida Masatsugu (dir.), Le Sanctuaire d'Ise, récit de la $62^{e}$ reconstruction, Bruxelles, Mardaga, 2015, p. 97.

9. Voir Soizik Bechetoille «La reconstruction du jardin du Palais de l'Est et la protection du paysage autour du site archéologique de l'ancienne capitale Heijō " actes du colloque Mutations paysagères et patrimoniales de la ville japonaise (octobre 2013), Paris, Collège de France, 2019.

10. Au Japon, le système de mesure traditionnel était fondé sur les proportions du corps humain, telles que le pied, qui donne le shaku 尺. Il s'est peu à peu normé, mais le shaku reste une unité de mesure de référence en restauration. 
elles aussi, issues de réemplois du site du palais de Fujiwara. Pourtant, les découvertes faites lors de l'excavation n'ont pas permis de trouver les pièces capables de déterminer la forme originelle complète. Comme la porte Suzaku du site palatial de Heian (actuelle ville de Kyōto 京都) comportait deux niveaux, on a assez naturellement procédé par analogie, quoique pour instruire complètement cet aspect, une équipe pluridisciplinaire a été formée, associant historiens, archéologues, architectes et ingénieurs. En 1965, une première tentative de restitution via la réalisation d'une maquette à l'échelle $1 / 10^{\mathrm{e}}$ a permis la mise au point de plans prévoyant l'emplacement de 18 poteaux de $7 \mathrm{~cm}$ de diamètre pour $53 \mathrm{~cm}$ de longueur pour le premier niveau. Après plusieurs essais, on obtint une maquette jugée capable de définir ce que la porte originale aurait pu être. D'une certaine façon, à l'image de la maquette du roman de Rohan, celle de la porte Suzaku portait en elle la promesse de la reconstruction in situ à l'échelle $1 / 1$. Près de trente ans plus tard, après une validation institutionnelle des hypothèses, le dessin des plans d'exécution commence.

La reconstruction de la porte Suzaku démarre en 1989 avec la reconstruction de la plate-forme. Cette dernière est achevée en 1992 et la reconstruction du bâtiment en lui-même débute en 1993. La porte fut entièrement reconstruite en cinq ans. D’après les données du site du Centre national de recherche pour les biens culturels de Nara (Nabunken 奈文研), la reconstruction de la porte Suzaku a exigé environ un millier de mètres cubes de bois - quantité nécessaire à l'érection de, cette fois, 18 poteaux de $70 \mathrm{~cm}$ de diamètre pour 5,3 mètres de longueur (coupés à Yoshino 吉野, préfecture de Nara).

Dans cet exemple, la maquette a notamment permis la mise en correspondance des données topographiques directement observables sur le terrain avec l'obligation d'extrapoler les élévations aux moyens de référents historiques plus ou moins proches. Les opérations qui consistent à produire une maquette connectent, par leur dimension active et leur proximité avec le geste de l'artisan qui, toujours, procède par reports de mesures, le maître charpentier qui respecte ces rapports de proportions traditionnels basés sur la longueur de l'entrecolonnement ou bien la hauteur du poteau par rapport au toit. Ces ratios fondent d'ailleurs l'art du kiwari et donnent les critères esthétiques d'appréciation d'un bâtiment et, dans le même temps, son style et son âge (ill. 1).

\section{La maquette comme objet de transmission des formes et des justes proportions}

Artisans de la construction en bois au Japon, les maîtres charpentiers étaient issus de familles qui avaient acquis une expertise et une renommée dans la construction de fortifications, de temples et de sanctuaires. À partir de l'époque de Heian (784-1185), la profession se développe, se structure et l'apprentissage de la charpenterie se fait dans le cadre d'une guilde ${ }^{11}$, de maîtres à élèves. La qualité de

11. La notion de guilde, qui vient de la tradition médiévale française, convient effectivement au cas des charpentiers japonais pour caractériser non seulement l'organisation autour d'activités communes mais aussi les règles et privilèges qui leur sont spécifiques. 


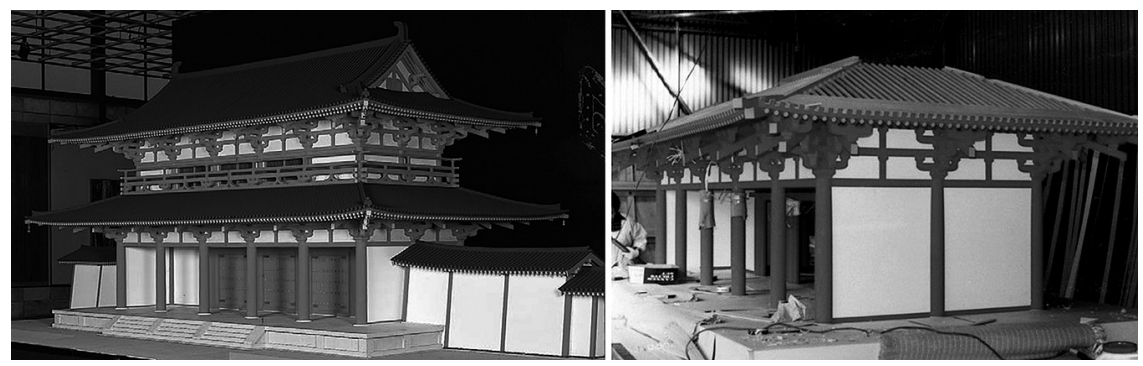

Ill. 1 : Maquette en bois de la porte de l'Oiseau rouge, 1965. Photographies issues du vol. 53, rapport de l'étude pour la reconstruction, centre de recherche pour les biens culturels de Nara 1994, 第 53 冊平城宮朱雀門の復原的研究奈良国立文化財研究所 1994, p. 153-154.

la transmission dans l'enseignement est un facteur parfois plus important que l'exécution même de l'ouvrage auquel un apprenti charpentier peut être assigné. Si autrefois, il fallait être introduit auprès d'un maître, la dynamique d'enseignement a aujourd'hui évolué et les entreprises proposent des stages où sont dispensés les enseignements de base. Dans ce contexte où l'approche traditionnelle prime, on pourrait penser que la maquette, à l'image des chefs-d'œuvre réalisés par les charpentiers compagnons du devoir en France, représente un "passage obligé " pour l'apprenti, une validation de son enseignement. Pourtant non, il ne s'agit pas de prouver sa virtuosité technique à travers un objet dont on admirerait la confection. La maquette, généralement réalisée par le maître daiku lui-même, est avant tout vecteur de savoir-faire, objet de transmission du respect absolu des proportions.

Chaque démontage d'édifice est une occasion unique pour comprendre l'évolution des techniques, des outils et du savoir-faire des charpentiers. Cela permet de vérifier la véracité de certains écris ou de certaines transmissions orales liées à l'histoire des lieux ou des édifices restaurés. La géométrie d'un bâtiment revêt donc une grande importance ${ }^{12}$. Ainsi, le modèle de la pagode préexiste à la pagode. Le galbe d'un toit n'est pas déterminé par l'évaluation du "bon angle" au regard d'un cas particulier : la silhouette générale du bâtiment est d'abord conçue dans une approche combinatoire qui donne priorité aux liens de réciprocité entre les différentes dimensions (de la partie au tout), de sorte que le problème du report n'est plus qu'une question de maniement d'outil, et non de calcul et de conversion. Ce fait explique notamment les qualités de longévité des formes à travers leur transmission : le galbe d'un toit ayant pour origine la formule fondamentale, on peut reproduire le schéma

12. L'architecture bouddhique japonaise est dominée par l'assimilation de styles architecturaux variés importés de Chine, par le filtre de la Corée. Les différentes phases d'importation au cours des siècles permettent à tous les styles de construction des six dynasties chinoises d'être représentés au Japon. Après l'arrivée du bouddhisme au VI ${ }^{\mathrm{e}}$ siècle, il s'agissait surtout de reproduire les bâtiments aussi fidèlement que possible. Si, peu à peu, les variations stylistiques et formelles se sont imposées jusqu'à former une typologie proprement japonaise quoique toujours marquée par les objets dont elle s'inspire, la transmission des formes dans le temps et l'espace reste clé pour comprendre cette architecture. 
indéfiniment et même extrapoler les parties disparues à partir d'un fragment ou d'un plan, comme dans le cas de l'élévation de la porte Suzaku.

L'art du kiwari s'est donc transmis à travers l'ouvrage de la maquette au sein des guildes de daiku, même si, vers 1608, la publication de manuels démocratise ces critères à l'ensemble des constructeurs de bâtiments, donnant parfois lieu à des applications hétérodoxes ou excessivement mathématisées. L'idée de kiwari montre combien l'appréciation esthétique prend sa source au niveau des pratiques de construction et ce qu'elles supposent en termes de métier et de logique de corporation. Par-là, on comprend l'importance de la maquette de projet, qui permet de faire reconnaître, au-delà de la seule maîtrise technique, celles des proportions.

\section{Les maquettes des "chantiers-écoles" des pagodes du Jison.in 慈尊院 et du Yakushiji 薬師寺}

Dans le cas de l'édification de la pagode du Jison.in, qu'on situe généralement au début des années 1500 mais qui demeure incertaine, des écrits du XIX ${ }^{\mathrm{e}}$ siècle affirment que l'édifice existait en 1624 dans la physionomie générale qu'on lui connaît aujourd'hui. Pourtant, la chute d'un grand pin survenue en 1740, les restaurations de 1880, de 1906, puis la pose du toit de cuivre de 1953 ont, toutes à leur tour, apporté un lot d'innovations techniques, parfois au sacrifice de la règle immuable de la reconstruction à l'identique. En 2010, la maquette a été commanditée par le prête du temple pour conserver une trace de la structure une fois la pagode reconstruite. L'idée était d'obtenir un "écorché " d'un côté et une maquette "finie " de l'autre à exposer dans le temple. Le maître charpentier commença donc l'ouvrage qu'il ne termina jamais pour des raisons de coûts. La maquette servit cependant d'objet d'étude aux charpentiers alors en formation sur le chantier. Elle a ensuite été rapportée dans les bureaux de la société à Chiga pour être présentée dans le parc d'exposition des maquettes de l'entreprise. Ainsi, après avoir été un objet de présentation au profit de la formation de jeunes charpentiers, elle devint un objet d'apparat qui sert de faire valoir à l'entreprise dans le cadre d'appels d'offres pour de futurs projets (ill. 2).

La maquette du " chantier-école " de la pagode du Yakushiji 薬師寺 ${ }^{13}$ réalisée en 2010 à partir des outils traditionnels du charpentier a, elle, servi de base aux premières hypothèses au moment même du démontage de l'ouvrage. Quand les charpentiers ont repéré des pièces qui prouvent que la structure, mise en difficulté par son propre poids, a nécessité très tôt (c'est-à-dire bien avant les premières grandes restaurations connues) une batterie de pièces supplémentaires destinées à redresser les toits et pallier leurs désordres structurels, les équipes durent prendre acte qu'outre l'abolition du mythe de l'état originel supposément parfait, la découverte de ces pièces ajoutées accuse des défauts de conception dès l'origine et inscrit le bâtiment

13. Ces maquettes ont été observées lors d'études de terrain menées par l'auteur entre 2009 et 2011. 

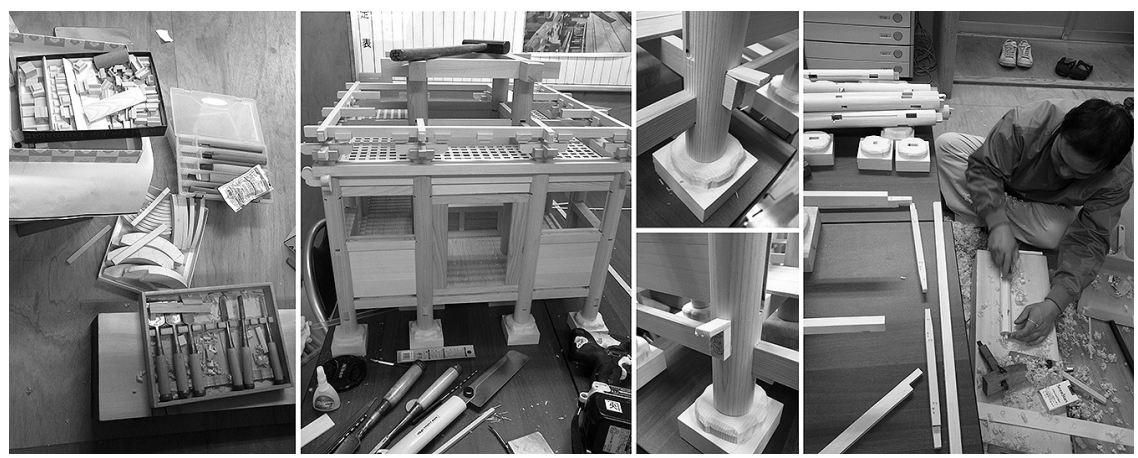

Ill. 2 : Maquette en bois du " chantier-école " de la pagode du Jison.in à Wakayama en cours de construction, 2009. (C) Photographies de l'auteur.

tout entier dans un mouvement d'amélioration permanent dont le chantier de 20092012 ne représente, finalement, qu'un segment. Il est alors intéressant de noter l'effort fourni par les charpentiers pour recopier ces «ates " et pour les intégrer au modèle réduit. La maquette $\mathrm{a}$, ici, vocation à montrer cette innovation (ill. 3).

Ainsi, dans le contexte des "chantiers-écoles ", la maquette sert à la saisie archéologique du bâti et permet de comprendre le choix de certains emboîtements es chantiers doivent également intégrer à leur répertoire formel ou à leur outillage méthodologique certaines concessions architecturales difficilement conciliables avec ce que la maquette ordinaire permet de modéliser. Ce que montre le chantier de restauration, c'est aussi l'importance de l'innovation dans la tradition. La reconstruction de la porte Suzaku et de la pagode du Jison.in ont nécessité l'utilisation de matériaux et de techniques modernes. Des tirants métalliques ont été ajoutés dans les deux cas, en toiture pour reprendre les charges et maintenir dans le temps un galbe impeccable. Des éléments antisismiques ont été ajoutés, là encore dans les deux cas, pour permettre aux bâtiments de mieux résister. Si les charpentiers japonais démontent avec beaucoup de respect les charpentes anciennes, ils savent aussi y lire les traces d'innovations laissées par leurs prédécesseurs dans les remontages successifs. Pour eux, il est également important et légitime de s'inscrire dans cette histoire constructive, et donc d'intégrer à la maquette ces éléments apparemment étrangers à la "perfection géométrique».

La maquette est l'objet par lequel se réactive la géométrie autrefois " parfaite " d'un bâtiment et celui qui permet au charpentier de s'y accoutumer. C'est aspect nous conduit bien au-delà du récit de Rohan qui, considérant la maquette comme objet de démonstration, négligeait la dimension didactique, mais il nous offre surtout l'occasion d'approcher les limites de ces objets d'étude et d'évoquer également ce qu'ils ne "montrent pas".

Même si la maquette reste, dans sa tradition constructive, fondamentalement liée à la recherche d'un équilibre entre les parties qui confere une valeur supérieure au bâtiment, et donc une légitimité au chantier, elle s'astreint bon an mal an à l'état 


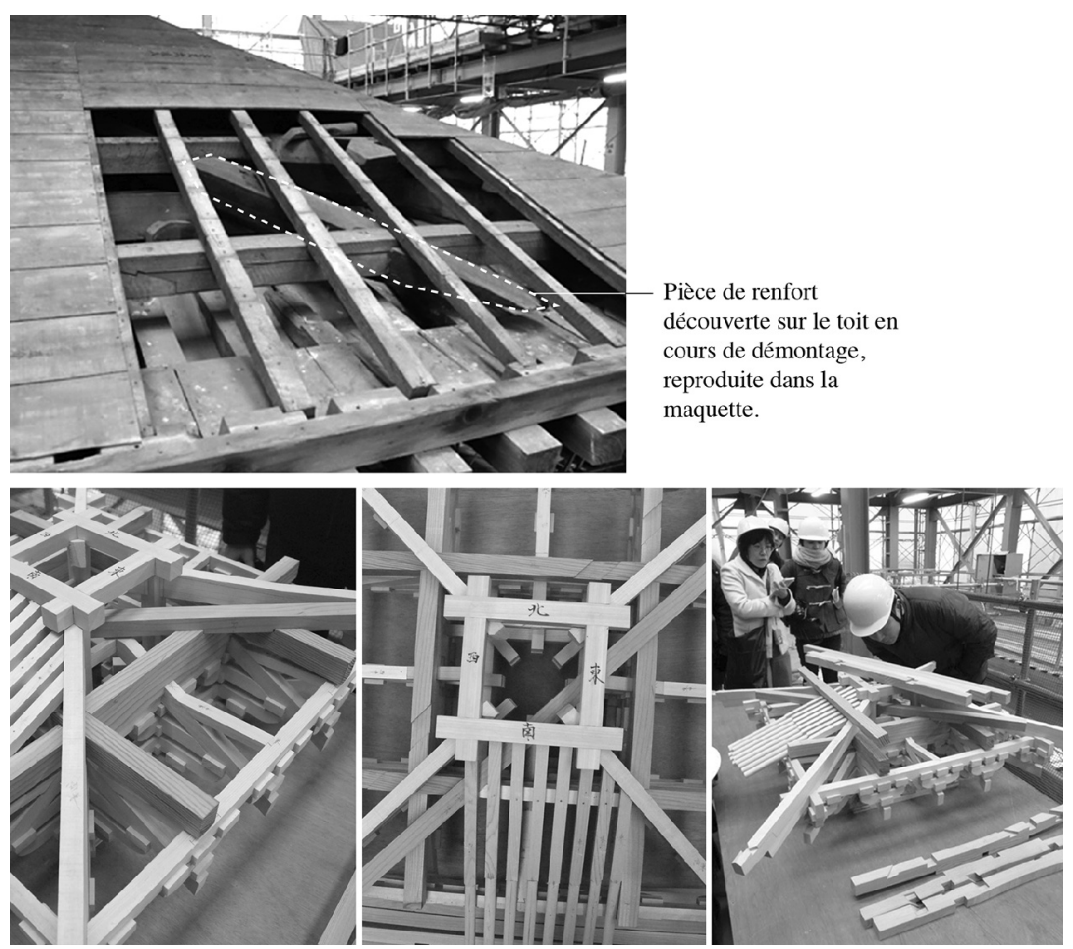

Ill. 3 : Détail du toit de la pagode du Yakushiji à Nara en cours de restauration et maquette en bois du "chantier-école", 2010. (C) Photographies de l'auteur.

de démonstration d'une architecture $100 \%$ bois, géométriquement idéale, soustraite à certaines contingences d'exécution. Elle est la reproduction du bâtiment en miniature, mais d'une certaine façon, elle ne peut représenter que son état premier supposé.

Ainsi, outre que l'échelle de la maquette (généralement $1 / 10^{\mathrm{e}}$ ) ne permet pas de modéliser les variations inhérentes aux mouvements du matériau ou à son vieillissement dans un environnement extérieur, l'imperfection du modèle originel ne peut pas être soupçonnée dans la maquette finie, quand bien même les artisans qui la confectionnent en ont effectivement pris la mesure et, dans certains cas, essayé d'en rendre compte par des ajouts dans le modèle réduit (qu'on laissera, à dessein, apparents). Pour autant, il convient de souligner les efforts effectués en faveur d'une maquette qui, soucieuse de pointer les "irrégularités " constructives, acquiert une valeur pédagogique qui s'applique également aux publics ou aux acteurs indirects du chantier (commanditaires, décideurs, financeurs...).

Même si les dimensions plutôt élevées des maquettes de charpentier permettent de vérifier sur pièce les hypothèses constructives qui concernent, par exemple, certains détails techniques, leur qualité de "double miniature " d'un bâtiment doit être nuancée par leur impuissance à restituer en un seul objet l'histoire d'un édifice 
et la complexité de ses multiples reconstructions. Habitude constructive autant qu'exigence académique, le "passage par la maquette " permet au charpentier de produire les conditions d'une réversibilité du bâtiment. En reproduisant l'édifice pièce par pièce, au fur et à mesure du démontage, le charpentier note, répertorie, consigne dans le bois les caractéristiques formelles réelles tout en recherchant, pardelà celles-ci et leurs éventuels accidents, la logique géométrique qui sous-tend l'ensemble de la construction. En cela, la fabrication de maquettes forme le charpentier à l'équilibrage savant des réalités tangibles avec les aspects immuables qui font le lit de l'histoire de l'architecture japonaise. Une attention particulière à l'exposition de ce «journal de bord en volume " que représente la maquette nous rapproche efficacement et sans effort des dispositifs muséographiques habituellement construits après coup.

Soizik BeChETOILLE-KaCZOROWSKI Architecte du patrimoine, PH.D, Ifpo (Institut français du Proche-Orient) 